\title{
Enhanced Photothermal Treatment Efficacy and Normal Tissue Protection via Vascular Targeted Gold Nanocages
}

\author{
Samir V. Jenkins, ${ }^{1 凶}$ Dmitry A. Nedosekin, ${ }^{2}$ Barry J. Shaulis, ${ }^{3}$ Tengjiao Wang, ${ }^{4}$ Azemat Jamshidi-Parsian, ${ }^{1}$ \\ Erik D. Pollock, ${ }^{3}$ Jingyi Chen, ${ }^{4}$ Ruud P.M. Dings, ${ }^{1}$ Robert J. Griffin ${ }^{1}$ \\ 1. Department of Radiation Oncology, University of Arkansas for Medical Sciences, Little Rock, AR \\ 2. Department of Otolaryngology and Phillips Classic Laser and Nanomedicine Laboratories, University of Arkansas for Medical Sciences, Little Rock, AR \\ 3. Trace Element and Radiogenic Isotope Lab, University of Arkansas, Fayetteville, AR \\ 4. Department of Chemistry and Biochemistry, University of Arkansas, Fayetteville, AR \\ $\triangle$ Corresponding author: Dr. Samir V. Jenkins, svjenkins@uams.edu, Department of Radiation Oncology, University of Arkansas for Medical Sciences, 4301 W. \\ Markham, Mail Slot \#771, Little Rock, AR 72205, USA \\ (C) Ivyspring International Publisher. This is an open access article distributed under the terms of the Creative Commons Attribution (CC BY-NC) license \\ (https://creativecommons.org/licenses/by-nc/4.0/). See http://ivyspring.com/terms for full terms and conditions.
}

Received: 2018.12.20; Accepted: 2019.03.14; Published: 2019.03.22

\begin{abstract}
A major challenge in photothermal treatment is generating sufficient heat to eradicate diseased tissue while sparing normal tissue. Au nanomaterials have shown promise as a means to achieve highly localized photothermal treatment. Toward that end, the synthetic peptide anginex was conjugated to Au nanocages. Anginex binds to galectin-1, which is highly expressed in dividing endothelial cells found primarily in the tumor vasculature. The skin surface temperature during a 10 min laser exposure of subcutaneous murine breast tumors did not exceed $43^{\circ} \mathrm{C}$ and no normal tissue damage was observed, yet a significant anti-tumor effect was observed when laser was applied $24 \mathrm{~h}$ post-injection of targeted nanocages. Untargeted particles showed little effect in immunocompetent, tumor-bearing mice under these conditions. Photoacoustic, photothermal, and ICP-MS mapping of harvested tissue showed distribution of particles near the vasculature throughout the tumor. This uptake pattern within the tumor combined with a minimal overall temperature rise were nonetheless sufficient to induce marked photothermal efficacy and evidence of tumor control. Importantly, this evidence suggests that bulk tumor temperature during treatment does not correlate with treatment outcome, which implies that targeted nanomedicine can be highly effective when closely bound/distributed in and around the tumor endothelium and extensive amounts of direct tumor cell binding may not be a prerequisite of effective photothermal approaches.
\end{abstract}

Key words: gold nanoparticles, photothermal therapy, galectin 1, targeting, tissue-mapping

\section{Introduction}

Gold nanomaterials are under intense investigation as a potential photothermal transducer for treatment of tumors and infections, with some systems having entered clinical trials (1-7). The highly uniform synthesis of these materials has been elucidated in recent decades (8), and the surface can be easily modified (9) to provide biocompatibility (10) and targeting of specific cells (11). Their large absorption cross-section makes them particularly enticing for photothermal treatment because a large effect can be achieved using a relatively low concentration of materials, and the materials themselves are typically found to be inert in biological systems $(12,13)$. Previous in vitro work in our lab and others has demonstrated that adding a targetingmoiety to promote binding to tumor cells appreciably enhances photothermal efficacy (14-16). For instance, we used the polydopamine-coated $\mathrm{Au}$ nanocages 
(AuNC@PDA) targeted to galectin-1 to achieve significant photothermal killing of cells without raising the bulk temperature in the well above $40{ }^{\circ} \mathrm{C}$ (17). Targeting was achieved using anginex (Ax), an antiangiogenic, synthetic 33-mer that has been shown to bind galectin-1 in tumors, particularly in the angiogenically active endothelium (18-21). Indeed, Ax has been shown to enable vascular targeting of liposomes (22). Other groups have targeted photothermally active materials to epidermal growth factor receptor (23), Her2 (24), CD-30 (25), carbonic anhydrase IX (26), and even biofilm-forming bacteria (27). These studies demonstrated marked increases in photothermal efficacy relative to their non-targeted analogues. Nanomaterial targeting has also demonstrated improvements in efficacy for drug delivery (28) and radiotherapy $(29,30)$.

Similar enhancements have been observed in vivo with a variety of particles (31), though in vivo systems add many additional layers of complexity including injection efficiency, animal and tumor model, size, and location. Au nanocages (AuNCs) have a high photothermal conversion efficiency, even amongst gold particle morphologies (14), and have been utilized for many photothermal studies in cancer. However, it is worth noting that in these studies the temperature of the tumor was either not measured, or reported to be well outside the traditional hyperthermic range $\left(<43{ }^{\circ} \mathrm{C}\right)$ e.g. $54^{\circ} \mathrm{C}$ (at the skin) (32), $60^{\circ} \mathrm{C}(33)$, or $70^{\circ} \mathrm{C}$ (34). Typically these studies employed a relatively high dose of nanoparticles and/or high laser power densities to achieve the reported effects, and it is unclear specifically where the particles were deposited in the tissue. The bulk heating effects on the tumor in these situations are ultimately similar to a cauterization or ablative event. Studies that use targeted particles, rather than simply relying on the enhanced permeability and retention effect, have shown improved therapeutic outcomes following photothermal treatment $(16,26,35,36)$.

Typically, particles are targeted to a specific molecule on the surface of the cancer cells, which still requires the particle to extravasate through the endothelium or gaps in the vessel wall prior to binding. Tumor vasculature, however, contains significant molecular distinctions from normal vasculature, including overexpression of galectin-1. In this report, AuNC@PDA-Ax was synthesized and characterized, and the construct was used for in vivo photothermal treatment of the 4T1 murine triple negative breast cancer model $(37,38)$. Particle localization and effect on endothelial cells were characterized. Additionally, serum stability, vascular pharmacokinetics, and tissue biodistribution were measured. A single dose of targeted particles and laser irradiation was applied and tumor growth was monitored as illustrated schematically in Figure $\mathbf{1 .}$ This treatment nearly doubled the time required for the tumor to reach a four-fold increase in volume. Most critically, optoacoustic tomography, mass analysis, and photoacoustic and photothermal microscopy were used to map the accumulation of the particle throughout the tumor tissue. These results present a new understanding of the required distribution and amounts of targeted nanocages sufficient for a marked therapeutic effect. The high locoregional heating from photothermal therapy can be precisely directed to the tumor which has clinically relevant implications since salvage therapy using such approaches has been clearly demonstrated to be of great value for patients with limited other options (39).
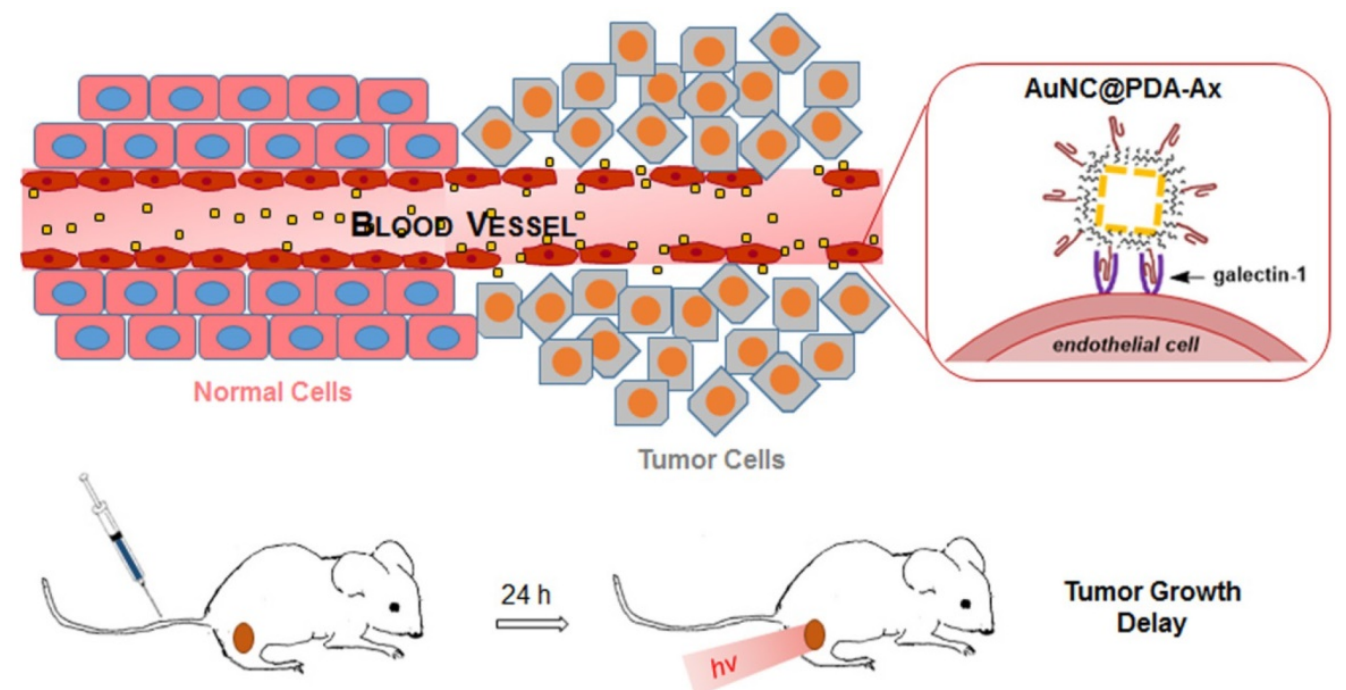

Tumor Growth Delay 


\section{Results and Discussion}

AuNCs were synthesized, coated with polydopamine (AuNC@PDA), and conjugated with anginex (AuNC@PDA-Ax) in accordance with our prior work (Figure S1). These nanocages had an edge length of $45.6 \pm 6.1 \mathrm{~nm}$, and an LSPR at $760 \mathrm{~nm}$ which shifted to $794 \mathrm{~nm}$ following Ax conjugation, which is included in Figure 2A. These values are consistent with our prior in vitro studies (17), and the shift in the LSPR position is largely the result of the change in refractive index due to the increasing quantity of the polydopamine heteropolymer. This LSPR position allows strong overlap with the laser used in therapeutic studies ( $808 \mathrm{~nm})$, and falls in the tissue transparent window, which allows light penetration of up to $1 \mathrm{~cm}$ through soft tissue.(40) The zeta potential (Figure 2B) changed from highly negative $(-40.0 \pm 2.2 \mathrm{mV})$ to positive $(+10.1 \pm 1.1 \mathrm{mV})$, which is to be expected, since at physiological $\mathrm{pH}, \mathrm{Ax}$ has a charge of $+4(41,42)$. Conjugation of the cationic peptide caused the zeta potential to change significantly, which is a straightforward method to confirm the conjugation. Our prior results showed a nonsignificant increase in the hydrodynamic diameter from $126 \pm 15 \mathrm{~nm}$ to $135 \pm 18 \mathrm{~nm}$ following conjugation, and indicated that on average $6 \times 10^{4} \mathrm{Ax}$ molecules were conjugated to each AuNC@PDA.

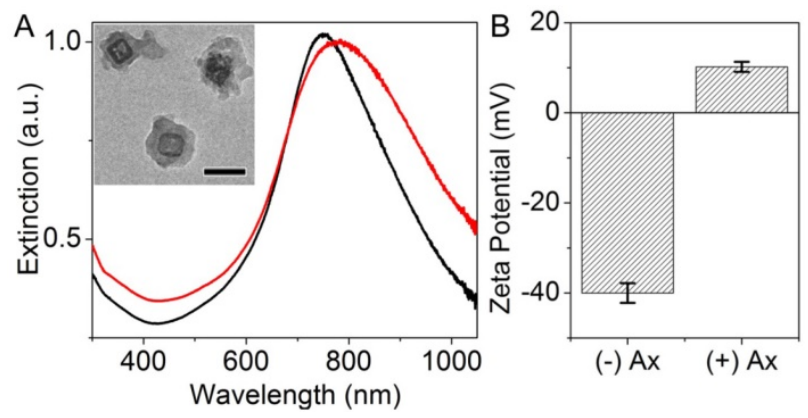

Figure 2. Characterization of materials. (A) Extinction spectra of AuNC@PDA before (black) and after (red) conjugation with anginex. The inset shows a TEM image of typical particles with $100 \mathrm{~nm}$ scale bar. (B) Zeta potential of the construct before and after conjugation with anginex.

The tube formation assay, an in vitro angiogenesis assay (43), was performed using the 2H11 mouse endothelial cell line (Figure 3A). The untreated (vehicle) and AuNC@PDA groups showed no significant difference in the ability to form higher structures. In contrast, both the $\mathrm{Ax}$ and the AuNC@PDA-Ax groups showed little tube formation after $2 \mathrm{~h}$, indicating that the biological function of $\mathrm{Ax}$ is retained following conjugation to AuNC@PDA. These results conform to earlier migration assays performed using this platform and generally indicate the AuNC@PDA-Ax has biological effects that are the result of the conjugated $\mathrm{Ax}$ rather than the particle itself. Additionally, photothermal microscopy (Figure 3B) was performed which showed that the Ax conjugated particles remain bound to the exterior of endothelial cells, rather than being internalized, as has been observed with Ax targeted liposomes (44), despite overnight incubation, which suggests that the proximity to the cells is sufficient to induce a potent photothermal effect.

Both AuNC@PDA and AuNC@PDA-Ax were found to have high stability in a serum mimetic solution (4\% BSA in PBS) (45) after $24 \mathrm{~h}$ without agitation (Figure 4A). The ability for nanoparticles to remain stable in serum is a critical consideration in development of nanomedicines, as many particles are prone to agglomerate and can change morphology and/or properties in the complex biological milieu of the bloodstream (46). Indeed the particle suspension was found to be more stable than the suspension in PBS or cell culture media (10\% serum), which we have previously reported, and the turbulent nature of flow throughout the bloodstream is expected to further prevent passive agglomeration, as they can be readily dispersed by inverting their container. Groups of Balb/c mice were subcutaneously inoculated with 4T1 tumor cells in the rear limb that were allowed to grow to $\sim 8-10 \mathrm{~mm}$ in diameter. At that time, particles were injected via tail vein and the photoacoustic signal in the ear vasculature was monitored for the following two hours (Figure 4B). The difference in signal intensity for the two systems is presumed to be due to variations in the sampling angle between the acoustic transducer, the ear vasculature, and the laser fiber. The pharmacokinetic profile was found to be bimodal for both constructs. Immediately following injection the PA signal increased by 8 orders of magnitudes, but dropped sharply over $\sim 15 \mathrm{~min}$, which yields a half-life $\left(t_{1 / 2} \alpha\right)$ of rough $3 \mathrm{~min}$, which reflects the time necessary for the bolus injection to disperse through the system volume. The depletion of signal slowed significantly over the remainder of the $2 \mathrm{~h}$ monitoring period. Due to signal heterogeneity and possible redistribution events after first pass kinetics, the determination of the $t_{1 / 2} \beta$ (elimination half-life) is somewhat complex, but it was estimated it to be on the order of $3 \mathrm{~h}$ for both non-conjugated and Ax-conjugated AuNC@PDA. At 24 hours after i.v. injection, mice were sacrificed and tissue harvested for ICP-MS (Figure 4C). The primary accumulation of the particles was in the spleen and liver as is common for nanomaterial distributions. Little accumulation was seen in the kidney as would be expected based on the size of the particle (47). The tumor saw an increase in particle accumulation from $22 \pm 8$ to $65 \pm 10 \mathrm{pg} \mathrm{Au}$ $\mathrm{g}^{-1}$ tissue following conjugation with $\mathrm{Ax}$. Ax also 
caused an apparent increase in accumulation in the lung, though no loss of body weight or abnormal behavior was observed prior to euthanasia. The increase in apparent lung uptake suggests that in subsequent developmental and translational investigations there will need to be careful attention paid to biodistribution and retention over time, which is beyond the scope our current work. The changes in accumulation are attributed to Ax, but the possibility that any peptide would similarly affect the transient distribution properties cannot be ruled out.

Photoacoustic microscopy was used to monitor the immediate accumulation of nanoparticles in the tumor (Figure 5A-D). For this study, a GFP expressing 4T1 was implanted in the mouse ear, which allowed for precise identification of the tumor location (Figure S2). The mouse was prepared for imaging and intravenous injection of AuNC@PDA-Ax was performed. No appreciable change in PA signal was observed in the normal tissue, while a $25-60 \%$ increase in PA signal was observed in the major vessel as well as two distinct tumor regions. Multispectral optical tomography (MSOT) was performed on rear limb tumors $24 \mathrm{~h}$ following i.v. administration of AuNC@PDA-Ax (0.1 pmol) (Figure 5E). This imaging modality, which relies on the photoacoustic signal of the AuNCs, demonstrated that the particles accumulated heterogeneously throughout the tumor, in particular accumulating in the periphery, which is commonly referred to as the viable rim of rapidly growing tumor tissue $(48,49)$. Appreciable signal also remained in the tail, which was the original site of injection, which suggests the possibility that some of the variability in nanomedicine experimental results could be due to the immediate arrest of particle dispersal in the tail vasculature, similar to radiotracer study experiences (50).

Following treatment with AuNC@PDA-Ax tumors were harvested, sectioned, and analyzed using several modalities. Laser ablation ICP-MS (LA-ICP-MS) was performed on these sections to map the distribution of $\mathrm{Au}$ throughout the tissue (Figure 6A, B) at higher resolution and with fewer artefacts than MSOT described above. Regions of high intensity were similarly found along the periphery, and pockets of high intensity were found throughout the tumor, some of which resemble vascular lumen structure. The glass slide itself showed minimal background signal and there were areas of the tumor that did not show high accumulation of the particles. In contrast, LA-ICP-MS of nontargeted AuNC@PDA (Figure S3) showed a heterogeneous distribution throughout the tumor. This heterogeneity, however, was notably different in that particles seemed to pool through the interstitium rather than localize in distinct regions. It should be noted that figures $\mathrm{S} 3$ and 6B are signal-normalized internally and cannot be quantitatively compared to each other. Serial sections were also imaged using photoacoustic microscopy (Figure 6C,D, S4) which showed a similar distribution along the periphery and heterogeneously throughout the tumor. Similar islands of CD31 positive cells were observed on the serial sections, which strongly suggests colocalization of the AuNC@PDA-Ax and endothelial cells as would be expected since particles arrive in the tumor vasculature, bind galectin-1, and extravasate to some degree. Silver can also be used to enhance the optical contrast of Au nanoparticles,(51) and double staining with silver enhancement and anti-CD31 (Figure S5) showed similar trends with non-targeted AuNC@PDA distributed throughout the tissue at low levels while AuNC@PDA-Ax showed more tendency to cluster near the CD31 positive regions. The PA system provides much greater resolution than the LA-ICP-MS system on a shorter timeframe. This same section was stained for nuclear content and imaged simultaneously using fluorescence and photothermal microscopy (Figure 6E). An appreciable colocalization was observed between the nuclear stain, autofluorescing regions of tissue, and AuNC PT signal. Specifically, the regions that lacked DAPI stain and tissue autofluorescence tended to be flanked with high photothermal signal, suggesting particle accumulation in the vasculature or perfused channels within the rapidly growing $4 \mathrm{~T} 1$ tumor.

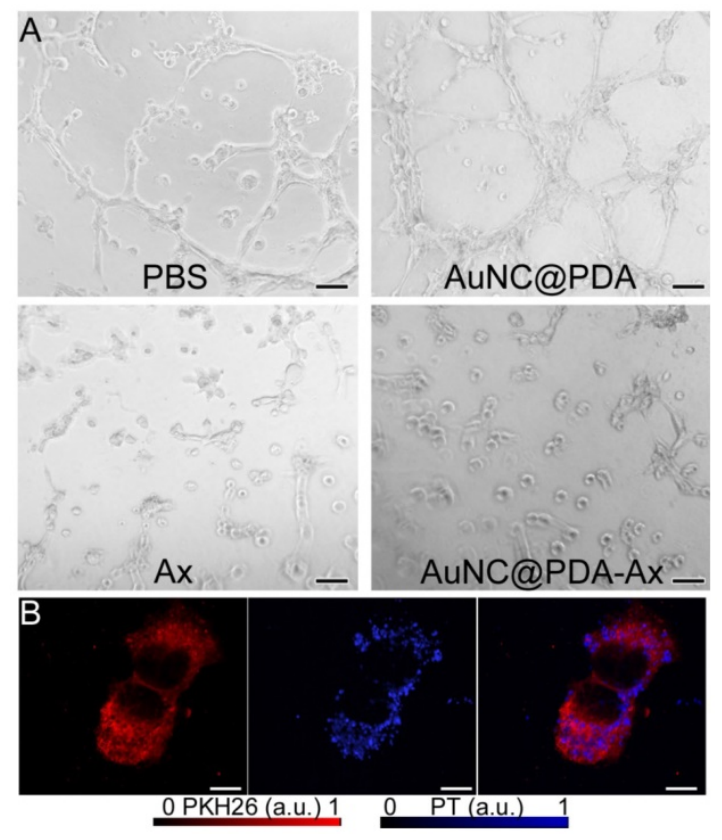

Figure 3. In vitro distribution and biological effect. (A) Representative images of tube formation using $2 \mathrm{H} 11$ cells after $2 \mathrm{~h}$ incubation with vehicle, AuNC@PDA, anginex, or AuNC@PDA-Ax. Scale bar is $100 \mu \mathrm{m}$. (B) Photothermal microscopy of $2 \mathrm{H} 11$ cells stained with PKH26 (orange) and AuNC@PDA-Ax (blue) and the two images merged. Scale bar is $10 \mu \mathrm{m}$. 

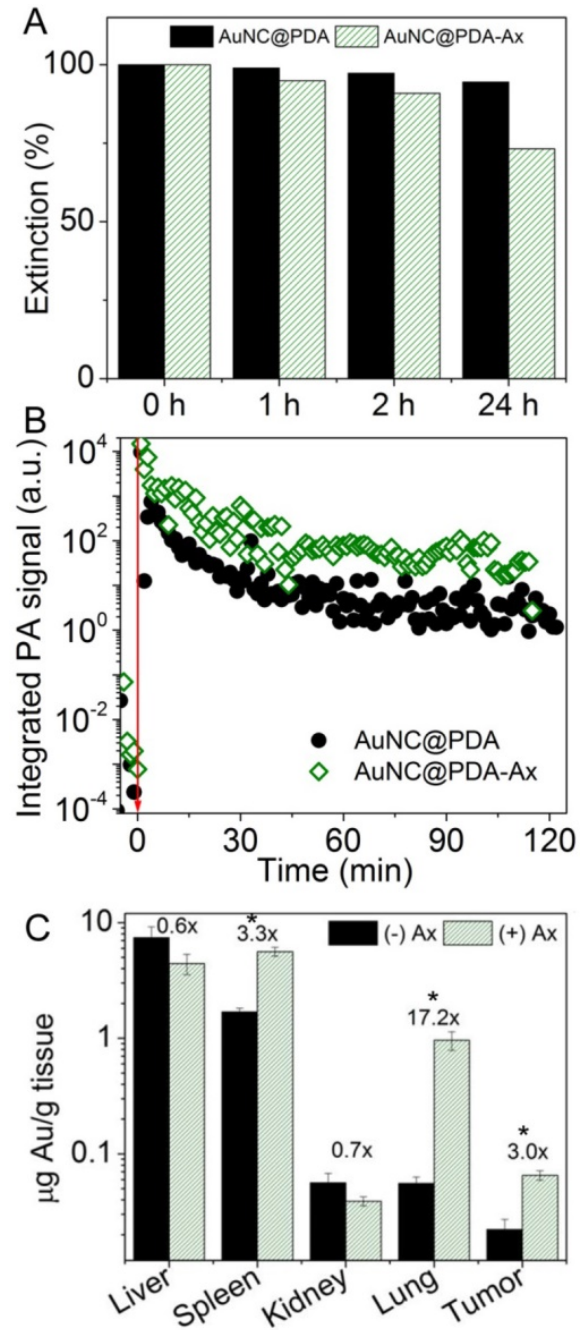

Figure 4. Stability, pharmacokinetics, and biodistribution of nanoconstructs. (A) Serum stability of the AuNC@PDA and AuNC@PDA-Ax construct over time. (B) Photoacoustic monitoring of ear vasculature immediately following tail vein injection of AuNC@PDA (circles) and AuNC@PDA-Ax (open diamonds). Points represent integrated PA signal over 1 min of monitoring. (C) ICP-MS of various organs 24 hours following tail vein injection of AuNC@PDA or AuNC@PDA-Ax. The numbers above the columns represent fold change. ${ }^{*}$ is $\mathrm{p}<0.05)$

After an i.v. injection of 0.1 pmol AuNC@PDA-Ax, the mice were treated with laser irradiation at $808 \mathrm{~nm}\left(1.2 \mathrm{~W} \mathrm{~cm}^{-2}\right)$ for 10 min using a spot size that completely covered the tumor area. Heating of the tissue was recorded every two minutes (Figure 7A), and there was a significant increase in temperature for the AuNC@PDA-Ax versus the laser alone. Using the non-targeted AuNC@PDA, a similar, though reduced increase in temperature was observed, which was attributed to the difference in total accumulation between targeted and non-targeted particles at $24 \mathrm{~h}$. Specifically, the average temperature at the surface was $4-6{ }^{\circ} \mathrm{C}$ higher in the nanoparticle treated animals regardless of targeting. The temperature during treatment (Figure 7B) was monitored to ensure this temperature did not exceed $43^{\circ} \mathrm{C}$, though increasing laser intensity could easily achieve significantly greater temperature changes. No evidence of tissue trauma was observed following the treatment described. Previous studies have targeted the VEGF receptor in a glioma using a window model, which showed evidence of vascular disruption following $6 \mathrm{~W} / \mathrm{cm}^{2}$ irradiation,(52) while another study used $\mathrm{CuS}$ nanoparticles targeting avb3 and 3 $\mathrm{W} / \mathrm{cm}^{2}$ to ablate tumors. These studies utilized greater laser fluence and focused on short term pathology, rather than the longer term tumor growth presented here.(53) However, we noted an initial inflammatory response in the overlying skin and muscle around the tumor after treatment that resolved over the course of the following week. Tumor growth was monitored for up to two additional weeks. The untreated, AuNC@PDA-Ax, and laser-alone groups had quadrupled in tumor volume after 6 days, which is consistent with historical data from our lab. The nanoparticle plus laser group, however, required 11 days to quadruple in size, nearly twice as long. Interestingly, the AuNC@PDA with laser group also showed a quadrupling of the tumor size in 8 days, despite temperature changes comparable to the AuNC@PDAAx group. These results suggest that the targeting aspect of our nanoconstruct specifically provided a significant therapeutic effect, which can be attributed to the close proximity between the particles and the membrane of the cells. It cannot be ruled out that the increased particle accumulation alone caused the photo-therapeutic effect observed. In fact, if judging by thermal dose alone as a predictor, the low bulk thermal dose achieved suggests there are likely additional mechanisms involved. This may include the role of $\mathrm{Ax}$ and biological effects induced in cells to which it binds, though the injected dose is roughly one-tenth of what has previously been found to be a therapeutic injection.(54) The initial increase in tumor volume observed is likely due to a transient inflammatory response to the treatment. It is also noteworthy that this was the result of a single treatment, rather than an extensive regimen of multiple sessions that would likely occur in the clinic. Future studies will consider the value of multiple injection and irradiation cycles as well as the value of multiple irradiation cycles following a single injection. No obvious toxicity was observed in the mice following AuNC@PDA or AuNC@PDA-Ax injection of 1 pmol of constructs (10 times greater than all studies presented). Weight loss was not observed, fur condition remained normal, and activity levels did not noticeably change. Hemolysis assay showed no significant lysis of the red blood cells relative to the PBS control (Figure S6). 

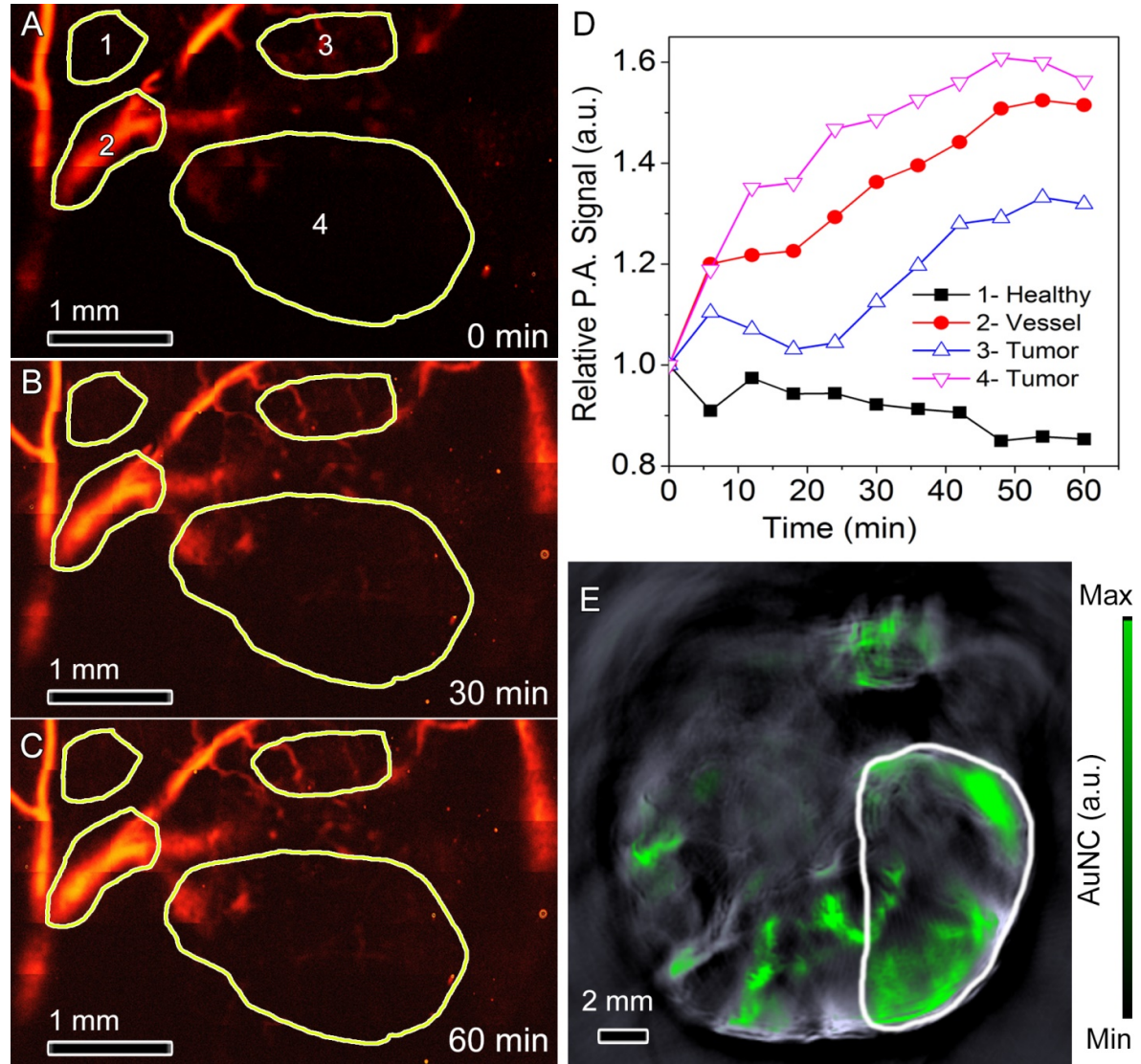

Figure 5. Photoacoustic monitoring of tumor accumulation. Photoacoustic mapping of an ear tumor (A) 0 , (B) 30, and (C) 60 min following i.v. injection of AuNC@PDA-Ax, with (D) quantification of the defined regions of (1) healthy tissue, (2) vessels, and $(3,4$,$) tumor tissue. Time points represent the accumulated P.A. signal of$ the region at the end of the 6 min collection time. (E) Multispectral optical tomography image of a slice of a similar hind limb tumor $24 \mathrm{~h}$ following i.v. injection of AuNC@PDA-Ax.
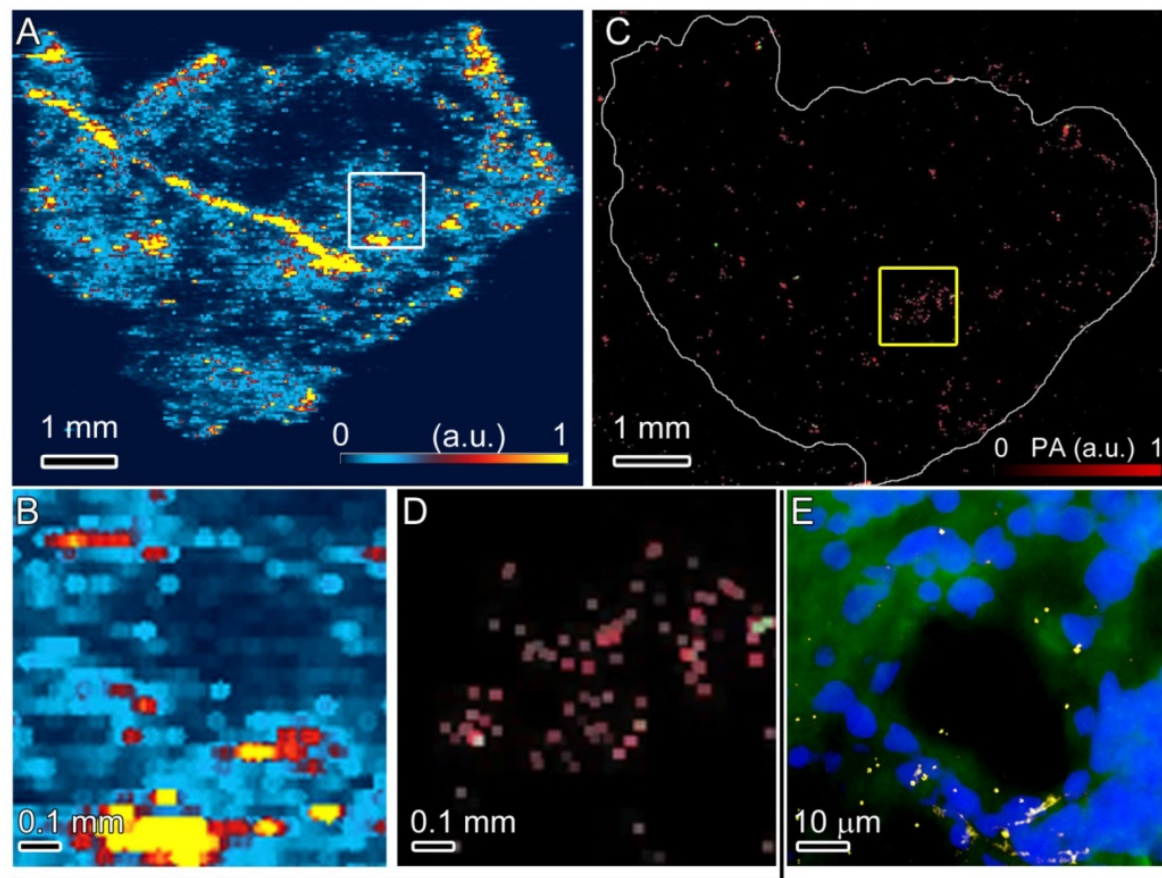

$\min$ Autofluorescence, a.u. $\max \min$

DAPI Fluorescence, a.u

PT signal, a.u.

Figure 6. Heterogeneous tumor distribution of AuNC@PDA-Ax. (A) Laser ablation ICP-MS mapping of a section of the AuNC@PDA-Ax treated tumor with blue representing low signal, red medium, and yellow high with the boxed area shown in (B); (C) wide field photoacoustic microscopy of a serial section of the same tumor where red represent photothermal signal and green/yellow represent artifacts (yellow being the overlap of red and green signal) with the boxed area shown in (D). Based on transmission images, the tumor is outlined in white for clarity; and (E) photothermal mapping of a representative region of the same section showing DAPI stain (blue), tissue autofluorescence (green), and photothermal signal from AuNCs (yellow). Intensity bars below the figure apply only to panel E. 

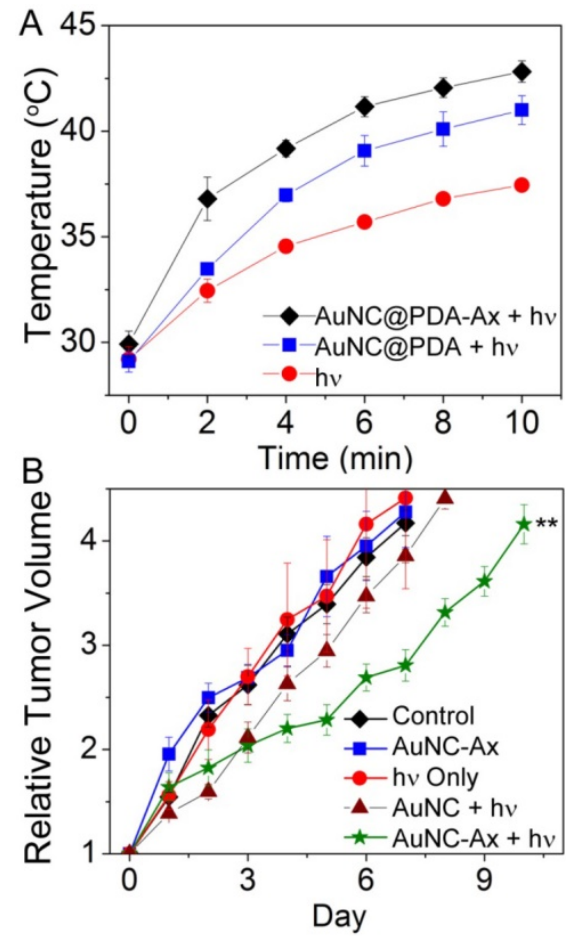

Figure 7. In vivo heating and tumor treatment using AuNC@PDA and AuNC@PDA-Ax. (A) Heating profile of the skin at over the tumor during laser irradiation with and without AuNC@PDA-Ax or AuNC@PDA. (B) Tumor growth curves ( $n=4-8$ mice per group) for mice treated with vehicle, 10 min laser irradiation, AuNC@PDA-Ax only, and the combination of either AuNC@PDA or AuNC@PDA-Ax and 10 min laser irradiation. (** is $p<0.01$ )

Importantly, the single irradiation was used to demonstrate proof of principle regarding the targeting capacity. While this study did not demonstrate complete eradication of the tumor, during clinical translation adjusting laser parameters and providing multiple rounds of laser irradiation can likely achieve this outcome. It is worth emphasizing that the laser dose was low in comparison with the majority of reports in the literature, which typically range from $4-20 \mathrm{~W} / \mathrm{cm}^{2}$, while studies using similar laser doses often use a pulsed laser or concurrent delivery of a chemotherapeutic for synergistic therapy.(55) Additionally, the use of interstitial lasers on small and medium sized lesions suggests this photothermal modality could be used even in deeper seated recurrent tumors or those with large volumes. One advantage of nanomaterials is the ability to serve as drug delivery platforms (56) and these materials can be engineered for thermal release of their cargo (45). They can enable multimodal therapies, and simultaneous diagnostic and therapeutic capacity has been demonstrated in many platforms. The relatively low temperature/time history reached during laser exposure would not be expected to have significant tumor killing effects if given using conventional hyperthermia applicators $(57,58)$, however there exists the possibility that these sublethal effects could affect membrane permeability, thereby affecting drug delivery,(59) or increase perfusion in the tumor, enabling multiple rounds of treatment to gradually penetrate deeper.(60) Various mapping methods illustrated the heterogeneous distribution of particles throughout the tumor, and these particles seem to cluster around vasculature, which can be attributed to Ax targeting of dividing vascular cells and the difficulty by which particles have in diffusing away from the blood supply (61-63). Since vascular cells serve as the support system for the tumor cells, (64) photothermal killing of these cells and subsequent inhibition of blood vessel function can lead to more effective therapy $(65,66)$. This study, similar to many biodistribution studies, detected a generally low overall accumulation of particles per gram of tumor tissue, however, potent photothermal effect in the tumor vasculature and surrounding perivascular tumor cells was sufficient to induce notable tumor response. This is an important finding, especially in that issues of drug or radiation resistance of tumor cells from prior treatment may be avoided and should be useful in development of this approach for salvage therapy in recurrent solid tumors.

\section{Conclusion}

This work demonstrates the potent in vivo photothermal capability of the AuNC@PDA system, and particular value is shown regarding the ability of Ax to appreciably increase the accumulation pattern and effects within the tumor. There were significant therapeutic responses despite a lack of cellular internalization or widespread uptake across the tumor interstitium. As such, it demonstrates the advantage of use of targeting agents aimed at neovascularization and/or the supporting tumor vasculature and suggests that this may be an effective alternative treatment approach in recurrent and/or therapy-resistant tumors. Tumor growth studies showed that the combination of the targeted nanomaterial and a single laser irradiation resulted in nearly doubling the time required for the tumor to quadruple in volume. These particles were found to have a reasonable pharmacokinetic half-life, and tumor accumulation was significantly enhanced as a result of the targeting agent. Considering the clear improvement observed in treatment response, further studies to increase particle accumulation and/or retention through new targeting moieties and perfusion enhancements appear warranted. Additionally, loading chemotherapeutics for drug delivery purposes and the ability of the AuNCs to be used as a radiosensitizer could add more potency to the photothermal effects observed here. 


\section{Experimental Section}

\section{Synthesis of PDA coated AuNCs (AuNC@PDA)}

The AuNCs were synthesized by the galvanic replacement reaction between $\mathrm{Ag}$ nanocubes and $\mathrm{HAuCl}_{4}$ as previously described (67) and fully detailed in the Supporting Information. AuNC@PDA was prepared by self-polymerization of dopamine on the surface of AuNCs under basic conditions in the presence of $\mathrm{O}_{2}$ as previously described (27). Briefly, 3 $\mathrm{ml}$ of $5 \mathrm{nM}$ AuNC aqueous suspension was diluted to $200 \mathrm{ml}$ using Tris-buffered saline $(20 \mathrm{mM}$ Tris and 100 $\mathrm{mM} \mathrm{NaCl}, \mathrm{pH}=9$ ) in a 250-ml, 3-neck, round-bottom flask. The reaction flask was briefly flushed with $\mathrm{O}_{2}$ and placed in a bath sonicator held at $4{ }^{\circ} \mathrm{C}$ with ice. Dopamine hydrochloride $(0.2 \mathrm{mmol}, 36.0 \mathrm{mg})$ was added to the flask, the vessel was sealed under $1 \mathrm{~atm}$ $\mathrm{O}_{2}$, and the mixture was sonicated throughout the reaction until the extinction peak of AuNC had red-shifted $\sim 30 \mathrm{~nm}(\sim 60 \mathrm{~min})$. The reaction was quenched by addition of $100 \mu \mathrm{L}$ concentrated acetic acid. After this reaction, the product was collected by centrifugation at $6000 \mathrm{rcf}$ for $10 \mathrm{~min}$, washed with $\mathrm{H}_{2} \mathrm{O}$ twice and recovered by centrifugation at 17,000 rcf for $10 \mathrm{~min}$ at $4{ }^{\circ} \mathrm{C}$. The AuNC@PDA was resuspended in $\mathrm{H}_{2} \mathrm{O}$ at a concentration of $6 \mathrm{nM}$ for characterization and future use. Transmission electron microscopy (TEM) images were acquired using a JEM-1011 (Jeol). Optical absorption spectra were recorded using a UV-vis spectrometer (Cary 50, Agilent). The elemental analysis was performed using an inductively coupled plasma mass spectrometer (ICP-MS; iCAP Q, Thermo Scientific, Waltham, MA). Zeta potential was measured in $1 \mathrm{mM} \mathrm{KCl}$ using a ZetaPALS instrument (Brookhaven).

\section{Anginex conjugation}

Anginex was synthesized and purified as previously described (20). Ax was conjugated to the surface of AuNC@PDA through the N-terminal amine and lysines by Michael addition to form AuNC@PDA-Ax (68). Briefly, 1 nM AuNC@PDA were dispersed in $1 \mathrm{ml}$ of $10 \mathrm{mM}$ borate buffer $(\mathrm{pH}=$ 9) and $100 \mu \mathrm{g} \mathrm{mL}-1$ of $\mathrm{Ax}$ was added to the solution. The reaction was allowed to proceed at $4{ }^{\circ} \mathrm{C}$ overnight. The conjugates were collected and washed three times with $\mathrm{H}_{2} \mathrm{O}$ by centrifugation at $19,000 \mathrm{rcf}$ for $5 \mathrm{~min}$ at $4{ }^{\circ} \mathrm{C}$. The conjugates were dispersed in $\mathrm{H}_{2} \mathrm{O}$ for future use and stored at $4{ }^{\circ} \mathrm{C}$. Colloidal stability was assessed by monitoring the absorbance at the LSPR maximum over time, in the absence of agitation, following dispersion of $100 \mathrm{pM}$ AuNC@PDA or AuNC@PDA-Ax in $\mathrm{H}_{2} \mathrm{O}$ and in 4 \% bovine serum albumin in PBS, which mimics serum.

\section{Tube formation assay}

Extracellular matrix Matrigel (Corning) was used to form a basement layer at bottom of 96 well plate (30 $\mu \mathrm{l}$ per well) following the manufacturer's instruction. Endothelial cells (2H11, $2 \times 10^{4}$ per well) were mixed to a final volume with PBS, $20 \mu \mathrm{g} \mathrm{mL}^{-1}$ Ax, or 20 pM AuNC@PDA or AuNC@PDA-Ax and seeded over the matrigel and incubated for $2 \mathrm{~h}$ to differentiate into capillary-like structures (tubes). Phase contrast images of formed tubes were acquired after 2 hours from several areas of each well.

\section{In vivo studies}

Female Balb/c mice averaging 6-8 weeks of age (Jackson Labs, Bar Harbor, ME, USA) were inoculated subcutaneously in the rear limb with $1 \times 10^{5} 4 \mathrm{~T} 1$ cells. After 8-10 days, tumors grew to a diameter of 8-10 $\mathrm{mm}$ at which time the animal was injected via tail vein with $100 \mu \mathrm{L}$ of $1 \mathrm{nM}$ (0.1 pmol) AuNC@PDA-Ax, AuNC@PDA, or PBS. Animals were imaged, treated, or euthanized $24 \mathrm{~h}$ after injection. For biodistribution studies, the liver, kidney, spleen, lungs, and tumor were harvested. For treatment, mice were anesthetized using isoflurane and irradiated for 10 minutes using a power density of $1.2 \mathrm{~W} \mathrm{~cm}^{-2}$ and a spot size of $14 \mathrm{~mm}$, which completely covered the tumor. Tumors were measured daily using calipers and the volume was calculated based on the equation $\mathrm{V}=\mathrm{a}^{*} \mathrm{~b}^{2} / 2$, where $\mathrm{V}$ is the volume and $\mathrm{a}$ and $\mathrm{b}$ are the length and width of the tumor. All studies were approved by the UAMS Institutional Animal Care and Use Committee.

\section{Inductively coupled plasma mass spectrometery}

Balb/c mice averaging 6-8 weeks of age (Jackson Labs, ME) were inoculated subcutaneously in the rear limb with $1 \times 10^{5} 4 \mathrm{~T} 1$ cells (vide supra). After 10 days the animals were injected intravenously via the tail with 0.1 pmol AuNC@PDA or AuNC@PDA-Ax (n=3 per group). Tumors were harvested $24 \mathrm{~h}$ with $18 \mathrm{M} \Omega$ $\mathrm{H}_{2} \mathrm{O}$ after injection and digested at $90{ }^{\circ} \mathrm{C}$ in $0.5 \mathrm{ml}$ $\mathrm{HNO}_{3}(99.999 \%)$ and $0.1 \mathrm{ml} \mathrm{H} \mathrm{O}_{2}(30 \%)$ overnight. After digestion, $0.25 \mathrm{ml} \mathrm{HCl}$ (99.999\%) was added; the solution was diluted to $15 \mathrm{ml}$ and filtered using a $70 \mu \mathrm{m}$ cell strainer (Fisher). ICP-MS was performed using iCAP Q (Thermo). Argon was used as a carrier gas at a flow rate of $1.05 \mathrm{ml} \mathrm{min}^{-1}$, and the fluid flow of $0.97 \mathrm{ml} \mathrm{min}^{-1}$. The matrix alone was run between samples to reduce sample carryover. Immediately prior to analysis, calibration standards between 0.1 and $250 \mathrm{ppb} \mathrm{Au}$ (1 $\mathrm{mg} \mathrm{mL}^{-1}$ stock, ULTRA Scientific) were prepared by serial dilution. 


\section{Photothermal Microscopy of Single Cells and Tissue Sections}

For photothermal microscopy (PTM) 2H11 cells were seeded into an 8-well chamber slide and incubated in complete medium overnight with AuNC@PDA-Ax, the following day PKH26 was added according to the manufacturer's instructions, and the sample was washed twice with PBS and fixed with $2 \%$ paraformaldehyde $\left(4{ }^{\circ} \mathrm{C}, 20 \mathrm{~min}\right)$. Tissue sections visualized using PTM microscope were prepared as described for ICP-MS and photoacoustic mapping (SI) and stained with DAPI according to the manufacturer's instructions.

\section{Photoacoustic Real-Time Quantification of Nanoparticles in Mouse Blood}

The photoacoustic fluorescence flow cytometry (PAFFC) system was used for real time monitoring of nanocages concentration in mouse blood after i.v. injection and was described elsewhere (69). Briefly, it was based on Nikon Eclipse E400 microscope platform (Nikon instruments, Inc., Melville, NY, USA). Photoacoustic signal was sensed using an acoustic transducer (model 6528101, 3.5 MHz, $4.5 \mathrm{~mm}$ in diameter; Imasonic Inc., Besançon, France) mounted over a heated $X Y$ positioning stage. PA signals were excited using $820 \mathrm{~nm}$ diode-pumped pulsed laser (maximal energy in the sample of $5 \mu \mathrm{J}$; pulse duration, $8 \mathrm{~ns}$; and pulse rate of $10 \mathrm{kHz}$, LUCE 820 , Bright Solutions, Italy). The laser was focused as a $5 \times 150 \mu \mathrm{m}$ line within mouse blood vessel. The transducer signal was amplified (preamplifier 5678; bandwidth, $200 \mathrm{kHz}$ - $40 \mathrm{MHz}$; gain $40 \mathrm{~dB}$; Panametrics NDT) and digitized (PCI-5124, 12-bit, 200 MSPS, National Instruments Inc.). All the data acquisition and analysis were performed using custom LabView based software. Tumor-bearing mice were anesthetized using isoflurane and positioned within the system to acquire a baseline reading. Then tail vein injection of AuNC@PDA or AuNC@PDA-Ax $(0.1 \mathrm{pmol})$ was performed and the PA signal in the ear vessel was acquired for 2 hours.

\section{Multispectral Optoacoustic Tomography (MSOT)}

The live imaging of mice was performed using the small-animal MSOT InVision256-TF (iThera Medical, Germany) (70). Irradiation is generated by a Class 1 laser with embedded Class 4 laser, capable of generating beams with wavelengths from $680-980 \mathrm{~nm}$, with a pulse length of $8 \mathrm{~ns}$, a maximum pulse energy of $120 \mathrm{~mJ}$, and a repetition rate of $10 \mathrm{~Hz}$. The geometrical arrangement of MSOT's 256-element transducer array enables detection of emitted ultrasonic waves ( $5 \mathrm{MHz}$ frequency) at a circumferential angle of 270 degrees and image acquisition rate of 10 $\mathrm{Hz}$. One day prior to imaging the entire circumference of the imaging area was depilated with Nair (Wal-Mart, Bentonville, AR) for two minutes, and the animal was thoroughly rinsed with tap water. The anesthetized mouse was placed in the supine position on the detachable holder docked at the mounting station in a thin, waterproof wrap made of transparent plastic. The mouse's limbs were stretched and fastened to prevent motion during monitoring. A thin layer of acoustic gel was placed around the mouse's body to facilitate acoustic coupling and visible air bubbles were removed. The holder along with the animal was submerged in the imaging chamber of the MSOT device, a bath filled with deionized water pre-heated to $34{ }^{\circ} \mathrm{C}$, and the temperature was allowed to equilibrate. Continuous delivery of isoflurane through a nose-cone in the holder ensured that the mouse remained anesthetized throughout the duration of the experiment. Continuous MSOT imaging was conducted in a transverse section through the mouse, consecutively, using wavelengths from 680-900 $\mathrm{nm}$ with 10 averages per position in $0.3 \mathrm{~mm}$ steps. The images acquired using MSOT were reconstructed using a linearregression algorithm, then put through multispectral processing using the pre-installed ViewMSOT3.8 software within the device, and a representative slice was selected.

\section{Abbreviations}

AuNC: gold nanocage; AuNC@PDA: gold nanocages coated with polydopamine; AuNC@PDA-Ax: gold nanocages coated with polydopamine conjugated to anginex; Ax: anginex; DAPI: 4',6-diamidino-2phenylindole; GFP: green fluorescent protein; ICPMS: inductively coupled plasma mass spectrometry; LA-ICP-MS: laser ablation inductively coupled mass spectrometry; MSOT: multispectral optical tomography; PA: photoacoustic; PAFFC: photoacoustic fluorescence flow cytometry; PBS: phosphatebuffered saline; PT: photothermal; PTM: photothermal microscopy.

\section{Acknowledgements}

This work was supported in part by NSF EPSCoR RIII Award 1457888, the Center for Microbial Pathogenesis and Host Inflammatory Responses grant P20GM103625 through the NIH National Institute of General Medical Sciences Centers of Biomedical Research Excellence (RPMD), Arkansas Breast Cancer Research Program, and Arkansas Biosciences Institute. We also thank V. Zharov and C. Huppell for technical advice. 


\section{Supplementary Material}

Supplementary figures and tables. http://www.ntno.org/v03p0145s1.pdf

\section{Competing Interests}

The authors have declared that no competing interest exists.

\section{References}

1. Dreaden EC, Alkilany AM, Huang X, Murphy CJ, El-Sayed MA. The golden age: gold nanoparticles for biomedicine. CHEM SOC REV. 2012;41(7):2740-79.

2. Zharov VP Mercer KE, Galitovskaya EN, Smeltzer MS Photothermal nanotherapeutics and nanodiagnostics for selective killing of bacteria targeted with gold nanoparticles. BIOPHYS J. 2006;90(2):619-27.

3. Huang X, Jain P, El-Sayed I, El-Sayed M. Plasmonic photothermal therapy (PPTT) using gold nanoparticles. LASER MED SCI. 2008;23(3):217-28.

4. Pilot Study of AuroLase(tm) Therapy in Refractory and/or Recurrent Tumors of the Head and Neck [Internet]. National Institute of Health. 2013. Available from: http://clinicaltrials.gov/ct2/show/NCT00848042.

5. Efficacy Study of AuroLase Therapy in Subjects With Primary and/or Metastatic Lung Tumors [Internet]. Available from: https://www.clinicaltrials.gov/ct2/show/NCT01679470.

6. Xia Y, Li W, Cobley CM, Chen J, Xia X, Zhang Q, et al. Gold Nanocages: From Synthesis to Theranostic Applications. ACCOUNTS CHEM RES. 2011;44(10):914-24.

7. Jenkins SV, Muldoon TJ, Chen J. Plasmonic Nanostructures for Biomedical and Sensing Applications. In: Xiong Y, Lu X, editors. Metallic Nanostructures: Springer International Publishing; 2015. p. 133-73.

8. Xia Y, Xiong Y, Lim B, Skrabalak SE. Shape-Controlled Synthesis of metal Nanocrystals: Simple Chemistry Meets Complex Physics. ANGEW CHEM INT EDIT. 2009;48:60-103.

9. Chen Y, Xianyu Y, Jiang X. Surface Modification of Gold Nanoparticles with Small Molecules for Biochemical Analysis. ACCOUNTS CHEM RES. 2017;50(2):310-9

10. Schöttler S, Becker G, Winzen S, Steinbach T, Mohr K, Landfester K, et al. Protein adsorption is required for stealth effect of poly(ethylene glycol)- and poly(phosphoester)-coated nanocarriers. NAT NANOTECHNOL. 2016;11:372.

11. Polo E, Collado M, Pelaz B, del Pino P. Advances toward More Efficient Targeted Delivery of Nanoparticles in Vivo: Understanding Interactions between Nanoparticles and Cells. ACS NANO. 2017;11(3):2397-402.

12. Fratoddi I, Venditti I, Cametti C, Russo MV. How toxic are gold nanoparticles? The state-of-the-art. NANO RES. 2015;8(6):1771-99.

13. Khlebtsov N, Dykman L. Biodistribution and toxicity of engineered gold nanoparticles: a review of in vitro and in vivo studies. CHEM SOC REV. 2011;40(3):1647-71.

14. Pattani VP, Tunnell JW. Nanoparticle-mediated photothermal therapy: A comparative study of heating for different particle types. LASER SURG MED. 2012;44(8):675-84.

15. Chen J, Wang D, Xi J, Au L, Siekkinen A, Warsen A, et al. Immuno Gold Nanocages with Tailored Optical Properties for Targeted Photothermal Destruction of Cancer Cells. NANO LETT. 2007;7:1318-22.

16. Li Z, Huang P, Zhang X, Lin J, Yang S, Liu B, et al. RGD-Conjugated Dendrimer-Modified Gold Nanorods for in Vivo Tumor Targeting and Photothermal Therapy. MOL PHARM. 2010;7(1):94-104

17. Jenkins SV, Nedosekin DA, Miller EK, Zharov VP, Dings RPM, Chen J, et al. Galectin-1-based tumour-targeting for gold nanostructure-mediated photothermal therapy. INT J HYPERTHER. 2018;34(1):19-29.

18. Thijssen VL, Barkan B, Shoji H, Aries IM, Mathieu V, Deltour L, et al. Tumor Cells Secrete Galectin-1 to Enhance Endothelial Cell Activity. CANCER RES. 2010.

19. Upreti M, Jyoti A, Johnson SE, Swindell EP, Napier D, Sethi P, et al. Radiation-enhanced therapeutic targeting of galectin-1 enriched malignant stroma in triple negative breast cancer. ONCOTARGET. 2016;7(27):41559-74.

20. Dings RP, Yokoyama Y, Ramakrishnan S, Griffioen AW, Mayo KH. The designed angiostatic peptide anginex synergistically improves chemotherapy and antiangiogenesis therapy with angiostatin. CANCER RES. 2003;63(2):382-5.

21. van der Schaft DW, Dings RP, de Lussanet QG, van Eijk LI, Nap AW, Beets-Tan RG, et al. The designer anti-angiogenic peptide anginex targets tumor endothelial cells and inhibits tumor growth in animal models. FASEB J. 2002;16(14):1991-3.

22. Brandwijk RJMGE, Mulder WJM, Nicolay K, Mayo KH, Thijssen VLJL, Griffioen AW. Anginex-Conjugated Liposomes for Targeting of Angiogenic Endothelial Cells. BIOCONJUGATE CHEM. 2007;18(3):785-90.

23. Melancon MP, Lu W, Yang Z, Zhang R, Cheng Z, Elliot AM, et al. In vitro and in vivo targeting of hollow gold nanoshells directed at epidermal growth factor receptor for photothermal ablation therapy. MOL CANCER THER. 2008;7(6):1730.
24. Au L, Zhang Q, Cobley CM, Gidding M, Schwartz AG, Chen J, et al Quantifying the Cellular Uptake of Antibody-Conjugated Au Nanocages by Two-Photon Microscopy and Inductively Coupled Plasma Mass Spectrometry. ACS NANO. 2009;4(1):35-42.

25. Qu X, Yao C, Wang J, Li Z, Zhang Z. Anti-CD30-targeted gold nanoparticles for photothermal therapy of L-428 Hodgkin's cell. INT J NANOMED. 2012;7:6095-103.

26. Chen Y, Bian X, Aliru M, Deorukhkar AA, Ekpenyong O, Liang S, et al. Hypoxia-targeted gold nanorods for cancer photothermal therapy. ONCOTARGET. 2018;9(41):26556-71.

27. Meeker DG, Jenkins SV, Miller EK, Beenken KE, Loughran AJ, Powless A, et al. Synergistic Photothermal and Antibiotic Killing of Biofilm-Associated Staphylococcus aureus Using Targeted Antibiotic-Loaded Gold Nanoconstructs. ACS INFECT DIS. 2016;2(4):241-50.

28. Jenkins SV, Nima ZA, Vang KB, Kannarpady G, Nedosekin DA, Zharov VP, et al. Triple-negative breast cancer targeting and killing by EpCAM-directed, plasmonically active nanodrug systems. NPJ PRECIS ONCOL. 2017;1(1):27.

29. Berbeco RI, Ngwa W, Makrigiorgos GM. Localized dose enhancement to tumor blood vessel endothelial cells via megavoltage X-rays and targeted gold nanoparticles: new potential for external beam radiotherapy. INT J RADIAT ONCOL BIOL PHYS. 2011:81(1):270-6.

30. Griffin RJ, Koonce NA, Dings RP, Siegel E, Moros EG, Brauer-Krisch E, et al. Microbeam radiation therapy alters vascular architecture and tumor oxygenation and is enhanced by a galectin- 1 targeted anti-angiogenic peptide. RADIAT RES. 2012;177(6):804-12.

31. Wang Y, Black KCL, Luehmann H, Li W, Zhang Y, Cai X, et al. Comparison Study of Gold Nanohexapods, Nanorods, and Nanocages for Photothermal Cancer Treatment. ACS NANO 2013;7(3):2068-77.

32. Liu Y, Ashton JR, Moding EJ, Yuan H, Register JK, Fales AM, et al. A Plasmonic Gold Nanostar Theranostic Probe for In Vivo Tumor Imaging and Photothermal Therapy. THERANOSTICS. 2015;5(9):946-60.

33. Chen J, Glaus C, Laforest R, Zhang Q, Yang M, Gidding M, et al. Gold Nanocages as Photothermal Transducers for Cancer Treatment. SMALL. 2010;6:811-7.

34. Maltzahn Gv, Park J-H, Agrawal A, Bandaru NK, Das SK, Sailor MJ, et al. Computationally Guided Photothermal Tumor Therapy Using Long-Circulating Gold Nanorod Antennas. CANCER RES. 2009;69(9):3892-900.

35. Piao J-G, Wang L, Gao F, You Y-Z, Xiong Y, Yang L. Erythrocyte Membrane Is an Alternative Coating to Polyethylene Glycol for Prolonging the Circulation Lifetime of Gold Nanocages for Photothermal Therapy. ACS NANO. 2014;8(10):10414-25

36. Lu W, Xiong C, Zhang G, Huang Q, Zhang R, Zhang JZ, et al. Targeted Photothermal Ablation of Murine Melanomas with Melanocyte-Stimulating Hormone Analog-Conjugated Hollow Gold Nanospheres. CLIN CANCER RES. 2009;15(3):876-86

37. Koonce NA, Quick CM, Hardee ME, Jamshidi-Parsian A, Dent JA, Paciotti GF, et al. Combination of Gold Nanoparticle-Conjugated Tumor Necrosis Factor-alpha and Radiation Therapy Results in a Synergistic Antitumor Response in Murine Carcinoma Models. INT J RADIAT ONCOL BIOL PHYS. 2015;93(3):588-96.

38. Koonce NA, Levy J, Hardee ME, Jamshidi-Parsian A, Vang KB, Sharma S, et al. Targeting Artificial Tumor Stromal Targets for Molecular Imaging of Tumor Vascular Hypoxia. PLOS ONE. 2015;10(8):e0135607.

39. Brooks ED Sun B, Feng $L_{\text {, }}$ et al. Association of long-term outcomes and survival with multidisciplinary salvage treatment for local and regional recurrence after stereotactic ablative radiotherapy for early-stage lung cancer. JAMA NETW OPEN. 2018;1(4):e181390.

40. Smith $\mathrm{AM}$, Mancini MC, Nie S. Bioimaging: second window for in vivo imaging. NAT NANOTECHNOL. 2009;4(11):710-1.

41. Griffioen AW, van der Schaft DW, Barendsz-Janson AF, Cox A, Struijker Boudier HA, Hillen HF, et al. Anginex, a designed peptide that inhibits angiogenesis. BIOCHEM J. 2001;354(Pt 2):233-42.

42. Dings RPM, Mayo KH. A Journey in Structure-Based Drug Discovery: From Designed Peptides to Protein Surface Topomimetics as Antibiotic and Antiangiogenic Agents. ACC CHEM RES. 2007;40(10):1057-65.

43. Nowak-Sliwinska P, Alitalo K, Allen E, Anisimov A, Aplin AC, Auerbach R, et al. Consensus guidelines for the use and interpretation of angiogenesis assays. ANGIOGENESIS 2018.

44. Upreti M, Jamshidi-Parsian A, Apana S, Berridge M, Fologea DA, Koonce NA, et al. Radiation-induced galectin-1 by endothelial cells: a promising molecular target for preferential drug delivery to the tumor vasculature. J MOL MED (BERL). 2013;91(4):497-506.

45. Jenkins SV, Srivatsan A, Reynolds KY, Gao F, Zhang Y, Heyes CD, et al. Understanding the interactions between porphyrin-containing photosensitizers and polymer-coated nanoparticles in model biological environments. J COLLOID INTERF SCI. 2016;461:225-31.

46. Jenkins SV, Qu H, Mudalige $\mathrm{T}$, Ingle TM, Wang R, Wang $\mathrm{F}$, et al. Rapid determination of plasmonic nanoparticle agglomeration status in blood. BIOMATERIALS. 2015;51(0):226-37.

47. Sonavane G, Tomoda K, Makino K. Biodistribution of colloidal gold nanoparticles after intravenous administration: Effect of particle size. COLLOID SURFACE B. 2008;66(2):274-80.

48. Liang $\mathrm{W}, \mathrm{Ni} \mathrm{Y}, \mathrm{Chen} \mathrm{F}$. Tumor resistance to vascular disrupting agents: mechanisms, imaging, and solutions. ONCOTARGET. 2016;7(13):15444-59. 
49. Chen M, Lei X, Shi C, Huang M, Li X, Wu B, et al. Pericyte-targeting prodrug overcomes tumor resistance to vascular disrupting agents. J CLIN INVEST. 2017;127(10):3689-701.

50. Lin JC, Song CW. Influence of vascular thermotolerance on the heat-induced changes in blood flow, pO2, and cell survival in tumors. CANCER RES. 1993;53(9):2076-80.

51. Choi CHJ, Alabi CA, Webster P, Davis ME. Mechanism of active targeting in solid tumors with transferrin-containing gold nanoparticles. Proceedings of the National Academy of Sciences. 2010;107(3):1235.

52. Day ES, Zhang L, Thompson PA, Zawaski JA, Kaffes CC, Gaber MW, et al. Vascular-targeted photothermal therapy of an orthotopic murine glioma model. Nanomedicine (London, England). 2012;7(8):1133-48

53. Cui L, Xiong C, Zhou M, Shi S, Chow DSL, Li C. Integrin av $\beta 3$-Targeted [64Cu]CuS Nanoparticles for PET/CT Imaging and Photothermal Ablation Therapy. BIOCONJUGATE CHEM. 2018;29(12):4062-71.

54. Dings RP, van der Schaft DW, Hargittai B, Haseman J, Griffioen AW, Mayo $\mathrm{KH}$. Anti-tumor activity of the novel angiogenesis inhibitor anginex. CANCER LETT. 2003;194(1):55-66.

55. Abadeer NS, Murphy CJ. Recent Progress in Cancer Thermal Therapy Using Gold Nanoparticles. J PHYS CHEM C. 2016;120(9):4691-716.

56. Srivatsan A, Jenkins SV, Jeon M, Wu Z, Kim C, Chen J, et al. Gold nanocage-photosensitizer conjugates for dual-modal image-guided enhanced photodynamic therapy. THERANOSTICS. 2014;4(2):163-74.

57. Griffin RI, Dings RP, Jamshidi-Parsian A, Song CW. Mild temperature hyperthermia and radiation therapy: role of tumour vascular thermotolerance and relevant physiological factors. INT J HYPERTHERM. 2010;26(3):256-63.

58. Griffin RJ, Lee SH, Rood KL, Stewart MJ, Lyons JC, Lew YS, et al. Use of arsenic trioxide as an antivascular and thermosensitizing agent in solid tumors. NEOPLASIA. 2000;2(6):555-60.

59. Hayat H, Friedberg I. Heat-induced alterations in cell membrane permeability and cell inactivation of transformed mouse fibroblasts. INT J HYPERTHERM. 1986;2(4):369-78.

60. Bagley AF, Scherz-Shouval R, Galie PA, Zhang AQ, Wyckoff J, Whitesell L, et al. Endothelial Thermotolerance Impairs Nanoparticle Transport in Tumors. CANCER RES. 2015

61. Dan M, Tseng MT, Wu P, Unrine JM, Grulke EA, Yokel RA. Brain microvascular endothelial cell association and distribution of a $5 \mathrm{~nm}$ ceria engineered nanomaterial. INT J NANOMED. 2012;7:4023-36.

62. Manzoor AA, Lindner LH, Landon CD, Park JY, Simnick AJ, Dreher MR, et al Overcoming limitations in nanoparticle drug delivery: triggered, intravascular release to improve drug penetration into tumors. CANCER RES. 2012;72(21):5566-75.

63. Kong G, Braun RD, Dewhirst MW. Characterization of the effect of hyperthermia on nanoparticle extravasation from tumor vasculature. CANCER RES. 2001;61(7):3027-32.

64. Denekamp J. Endothelial cell proliferation as a novel approach to targeting tumour therapy. BRIT J CANCER. 1982;45(1):136-9.

65. Horsman MR. Enhancing the radiation response of tumors but not early or late responding normal tissues using a vascular disrupting agent. ACTA ONCOL. 2017;56(11):1634-8.

66. Horsman MR, Siemann DW. Pathophysiologic effects of vascular-targeting agents and the implications for combination with conventional therapies. CANCER RES. 2006;66(24):11520-39.

67. Skrabalak SE, Au L, Li X, Xia Y. Facile synthesis of Ag nanocubes and Au nanocages. NAT PROTOC. 2007;2(9):2182-90.

68. Ye Q, Zhou F, Liu W. Bioinspired catecholic chemistry for surface modification. CHEM SOC REV. 2011;40(7):4244-58.

69. Nedosekin DA, Sarimollaoglu M, Foster S, Galanzha EI, Zharov VP, editors. Photoacoustic-fluorescence in vitro flow cytometry for quantification of absorption, scattering and fluorescence properties of the cells. SPIE BIOS 2013: 8581: 858141

70. Khanal A, Ullum C, Kimbrough CW, Garbett NC, Burlison JA, McNally MW, et al. Tumor targeted mesoporous silica-coated gold nanorods facilitate detection of pancreatic tumors using Multispectral optoacoustic tomography. NANO RES. 2015;8(12):3864-77. 